\title{
Using the Biopsychosocial Model for Identifying Subgroups of Detained Juveniles at Different Risk of Re-offending in Practice: a Latent Class Regression Analysis Approach
}

\section{Esther Laura de Ruigh}

Amsterdam UMC - Locatie VUMC: Amsterdam UMC Locatie VUmc

\section{Samantha Bouwmeester}

Erasmus University Rotterdam

\section{Arne Popma}

Amsterdam UMC - location VUmc

Robert Vermeiren

LUMC

\section{Lieke van Domburgh}

Amsterdam UMC - Location VUmc

Lucres MC Jansen ( $\sim$ L.nauta-jansen@amsterdamumc.nl)

Amsterdam UMC - Locatie VUMC: Amsterdam UMC Locatie VUmc https://orcid.org/0000-0001-84754050

Research article

Keywords: juvenile offenders, reoffending, latent class regression, subgroups, neurobiology, risk assessment

Posted Date: January 23rd, 2021

DOl: https://doi.org/10.21203/rs.3.rs-149321/v1

License: (c) (i) This work is licensed under a Creative Commons Attribution 4.0 International License. Read Full License 


\title{
Using the biopsychosocial model for identifying subgroups of detained juveniles at different risk of re-offending in practice:
}

\section{a latent class regression analysis approach}

\author{
E.L. de Ruigh ${ }^{a}$, S. Bouwmeester ${ }^{b}$, A. Popma ${ }^{a}$, R. Vermeiren ${ }^{c}$, L. van Domburgh ${ }^{a, d}$, L.M.C. Jansen ${ }^{a}$ \\ a Department of Child and Adolescent Psychiatry \& Psychosocial Care, Amsterdam UMC -VUmc, Amsterdam, \\ The Netherlands \\ ${ }^{b}$ Erasmus University Rotterdam, Rotterdam, The Netherlands \\ ${ }^{c}$ Department of Child and Adolescent Psychiatry, Leiden University Medical Center, Oegstgeest, The \\ Netherlands \\ d Pluryn-Intermetzo, Lelystad, The Netherlands
}

Corresponding author:

Lucres Nauta-Jansen PhD, Head research section Youth at risk

Principal Investigator

Dept of Child and Adolescent Psychiatry \& Psychosocial Care

AmsterdamUMC-VUmc

E-mail: I.nauta-jansen@amsterdamumc.nl

Visiting address: Meibergdreef 5 | 1105 AZ | Amsterdam

Postal address: Postbus 22000 | 1100 DD Amsterdam

Phone: 06 - 21654656 or Secretariat Section Youth at risk: 020- 8901545 


\section{Abstract}

Background: Juvenile delinquents constitute a heterogeneous group, which complicates decision-making based on risk assessment. Various psychosocial factors have been used to define clinically relevant subgroups of juvenile offenders, while neurobiological variables have not yet been integrated in this context. Moreover, translation of neurobiological group differences to individual risk assessment has proven difficult. We aimed to identify clinically relevant subgroups associated with differential youth offending outcomes, based on psychosocial and neurobiological characteristics, and to test whether the resulting model can be used for risk assessment of individual cases.

Methods: A group of 263 detained juveniles from juvenile justice institutions was studied. Latent class regression analysis was used to detect subgroups associated with differential offending outcome (recidivism at 12 month follow-up). As a proof of principle, it was tested in a separate group of 76 participants whether individual cases could be assigned to the identified subgroups, using a prototype 'tool' for calculating class membership.

Results: Three subgroups were identified: a 'high risk - externalizing' subgroup, a 'medium risk - adverse environment' subgroup, and a 'low risk - psychopathic traits' subgroup. Within these subgroups, both autonomic nervous system and neuroendocrinological measures added differentially to the prediction of subtypes of reoffending (no, non-violent, violent). The 'tool' for calculating class membership correctly assigned $92.1 \%$ of participants to a class and reoffending risk.

Conclusions: The LCRA approach appears to be a useful approach to integrate neurobiological and psychosocial risk factors to identify subgroups with different re-offending risk within juvenile justice institutions. This approach may be useful in the development of a biopsychosocial assessment tool and may eventually help clinicians to assign individuals to those subgroups and subsequently tailor treatment based on their re-offending risk.

Key words: juvenile offenders, reoffending, latent class regression, subgroups, neurobiology, risk assessment 
Using the biopsychosocial model for identifying subgroups of detained juveniles at different risk of re-offending in practice: a latent class regression analysis approach

\section{List of abbreviations:}

ANS - autonomic nervous system

HR - heart rate

PNS - parasympathetic nervous system

HRV - heart rate variability

RSA - respiratory sinus arrhythmia

SNS - sympathetic nervous system

PEP - pre- ejection period

ECG - electrocardiography

ICG - impedance cardiography

VU-AMS - VU- Ambulatory Monitoring System

SES - socio-economic status

YPI-s - Youth Psychopathic Traits Inventory-Short version

PT - psychopathic traits

BPM-Y - Brief Problem Monitor - Youth

ATMQ - Adolescent Treatment Motivation Questionnaire

CTQ-SF - Childhood Trauma Questionnaire - short form

LCRA - latent class regression analysis

PPV - positive predictive value

NPV - negative predictive value 


\section{Introduction}

Antisocial youth are known to be a heterogeneous group, for example in relation to psychiatric disorders and mental health needs (e.g. 1). This heterogeneity bears relevance for development, course, and prognosis of antisocial behavior (2). Research focusing on more accurate prediction of reoffending is vitally important, especially as reoffending rates of juvenile delinquents in the Netherlands have shown a smaller decline over recent years than e.g. adult offenders (3). Distinguishing more homogeneous subgroups of juvenile offenders may help in better predicting and eventually preventing future delinquent behavior.

Previous research has focused on defining homogeneous subgroups of juvenile delinquents, based on offender characteristics such as environmental factors, psychological and individual characteristics, personality traits, or combinations thereof $(2,4-8)$. Frequently, clusters are found that vary in terms of severity, for example in terms of problem behavior (e.g. 6, 9, 10, 11). In addition, integration of risk factors for antisocial behavior, problem behavior and mental health problems, substance use, and psychopathic traits, often prove important when clustering detained juveniles. $(2,6,8,9)$. Several studies have shown that identifying subgroups is relevant for identifying recidivism risk. For example, in serious juvenile offenders a subgroup of violent property offenders, who were also identified as the most serious repeat offenders, had the highest number of risk factors (12). Another study showed that most chronic and severe offenders were adolescents with all three psychopathy dimensions present (13). As for severity of recidivism, a distinction is made between non-violent and violent recidivism. Factors predictive of recidivism have been shown to be different for violent young offenders (14). For different subgroups, this information can provide different focus regarding (allocation to) intervention (see e.g. $6,15)$. The information gained from subtyping can therefore also inform treatment.

Notwithstanding the fact that previous studies yielded clinically relevant clusters, they were usually based on psychosocial offender characteristics. Although these factors have traditionally attracted the most attention in explaining antisocial behavior, the growing body of research shows that neurobiology is increasingly seen as a valuable additional aspect to understanding antisocial behavior. Several researchers argue in favor of a biopsychosocial framework (16-18), which states that interactions between psychosocial and neurobiological factors influence the development of antisocial behavior $(17,19,20)$. Furthermore, developmental crime prevention programs that recognize both the importance of the environment and biology were shown to reduce 
crime (21). However, neurobiological measures are not commonly used in risk assessment and treatment allocation in clinical practice.

Research has identified several potential peripheral biomarkers of antisocial behavior that can be relatively easily applied in clinical practice, such as heart rate (HR), cortisol, and testosterone (e.g. 22, 23, 24). HR is an indicator of activity of both the parasympathetic (PNS) as well as the sympathetic (SNS) branch of the autonomic nervous system (ANS). Meta-analyses showed that HR in rest and reactivity to a stressor is a consistent biological correlate of antisocial behavior in juveniles $(22,23)$. Longitudinally, low resting HR at age 15 was associated with criminal status at age 24 (25), and persistent delinquent behavior in a subsample from this population (26). In several studies, lower HR (rest and reactivity), and aberrant PNS (re)activity, were found to be predictive of increases in delinquent behavior and reoffending (27-30). With regard to SNS functioning, poor autonomic fear conditioning at age 3 was associated with criminal offending 20 years later (31). Furthermore, diminished SNS reactivity came forward as risk factor for externalizing problems (32). The steroid hormones cortisol and testosterone (easily measured in saliva) have also been associated with antisocial behavior (e.g. 33). Although mixed results have been found with respect to cortisol levels and antisocial behavior, a negative relationship between decreased cortisol (re)activity and antisocial behavior is reasonably consistent (34). A meta-analysis showed a positive relationship between testosterone and aggression, however not all studies find this link (35). Growing evidence shows cortisol and testosterone are interdependent in their influence on antisocial behavior (e.g. 36), and should therefore be studied together.

The use of neurobiological measures in practice however still faces major challenges, as inferences on an individual level cannot be made. As Moffitt, order (37) stated, there is no consensus on when biomarker levels are clinically significantly aberrant. Moreover, predictive values of single neurobiological measures are low at best $(38,39)$.Therefore, in the current study, we use the biopsychosocial model and integrate neurobiological and psychosocial measures to identify clinically relevant subgroups of detained juveniles associated with youth offending outcomes. By using an advanced form of latent class analysis, namely latent class regression analysis (LCRA; as advocated in 40), it is explored whether groups can be identified that differ with respect to various psychosocial and neurobiological characteristics, and their relationship with reoffending. Moreover, to test 
whether individual cases can be correctly assigned to the subgroups and reoffending risk, we used a prototype 'tool' for calculating class membership based on the LCRA model.

\section{Method}

\section{Participants}

Participants were 393 detained male juveniles between the age of 12 and 24, recruited from five juvenile justice institutions in the Netherlands. Exclusion criteria were unwillingness or inability to sign informed consent, insufficient command of the Dutch language, cardiac problems that would interfere with the heart rate measurements (e.g. arrhythmia and asthma), and inability to understand instructions and questionnaires, which was brought to our attention by institution staff. Recidivism rates within 12 months after release were obtained through judicial files. Upon follow-up, two participants were deceased, and for 128 participants the follow-up window was too short ( $<18$ months) to reliably determine recidivism. For this reason, these participants were excluded in the latent class regression analysis. The total sample for latent class regression analysis included 263 detained male juveniles (mean age $=18.67, S D=1.66$ ). The data of the participants with insufficient follow-up duration $(n=128)$, that were excluded from the latent class regression analysis, were used to evaluate the external validity of the model. For 52 of these 128 participants there were missing data, which allowed the model to be tested on 76 participants.

All participants, and when under the age of 18 also parents/caregivers, signed an informed consent document before participation. This study was approved by the Ethics Committee at the University of Amsterdam, and performed in accordance with the ethical standards described in the 1964 Declaration of Helsinki.

\section{Measures}

Questionnaires concerning demographic characteristics provided information regarding age, ethnicity, socioeconomic status (SES), ethnicity, and presence of criminal peers. To assess ethnicity, participants were asked in which country they and their (biological) mother and father were born. Ethnicity was determined by the place of birth of parents (e.g. non-western, when one or both parents were born in a non-western country). In order to estimate SES, participants were asked to provide the postal code of the address where they had lived 
the longest during their youth. This was then recoded into low, middle or high SES based on data from Statistics Netherlands (CBS). To obtain an indication of the amount of criminal friends, participants were asked whether they have friends who are sometimes involved in crime (yes/no).

The Youth Psychopathic Traits Inventory-Short version (YPI-s; 41) is a self-report measure to assess psychopathic-like traits in adolescents. It consists of three subscales measuring the interpersonal, affective and behavioral dimensions of psychopathic traits. The YPI-s consists of 18 items with a 4-point Likert scale ranging from 1 (does not apply at all) to 4 (applies very well). Higher YPI-s scores on any of the scales indicate a likelihood of a higher degree of psychopathic traits on that dimension. Research has supported the YPI three factor structure and has shown that the instrument is internally consistent (42). This measure has been shown to identify detained youths with high levels of psychopathic-like traits (43).

The Brief Problem Monitor - Youth (BPM-Y; 44) measures potential problems in youths' functioning along three dimensions: Internalizing, Externalizing and Attentional Problems. The BPM is a rating instrument for monitoring children's functioning and responses to interventions (RTIS). The items are drawn from the Child Behavior Checklist for Ages 6-18 (CBCL/6-18), Teacher's Report Form (TRF), and Youth Self-Report (YSR) (45). Each item is rated 0 (not true), 1 (somewhat true), or 2 (very true). Higher BPM-Y subscale scores are indicative of more problems along the relevant dimensions. Higher BPM-Y total scores are indicative of more overall problems. In the current study, only the youth self-report version of the BPM was used.

The Adolescent Treatment Motivation Questionnaire (ATMQ) is a self-report instrument measuring treatment motivation. It was derived from the MTQ, based on the TTM of Prochaska and DiClemente (46). The ATMQ consists of 11 items measuring the active phase of treatment motivation as a single construct, and is currently in use as part of the Dutch Routine Outcome Monitoring project in secure residential youth care. A 3-point answering scale with thumbs pictures is used for better comprehension. Items are rated untrue, somewhat true, or true. Higher scores on the scale for treatment motivation indicate greater treatment motivation. Internal consistency reliability was good (Cronbach's alpha .84) for a sample of adolescents in Dutch secure juvenile facilities (47). 
The short form of the Childhood Trauma Questionnaire (CTQ-SF; 48) is a standardized, retrospective 28-item self-report inventory that measures the severity of different types of childhood trauma, producing five clinical subscales: Emotional Abuse, Physical Abuse, Sexual Abuse, Emotional Neglect and Physical Neglect. CTQ items are given a score of 1 through 5 on a five-point Likert type scale, ranging from 1 (never true), to 5 (very often true). Higher scores on the scales mean higher levels of whatever that scale represents. The CTQ is validated for adolescent psychiatric patients (49) and male and female street-youth (50). The original CTQ consists of 70 items, but the version with 28 items is also validated for multiple populations (48). Item 24 has been removed because experience and research showed a low validity of this item.

An indication of substance use frequency was obtained by means of self-reports of drug use. Participants were asked on an 11-item rating scale how many times they had used various drugs (e.g., alcohol, marijuana, ecstasy) in the last month and in their lifetime. Response choices increased in intervals of 10 (e.g. $1=$ never used, 2=110 times, $3=11-20$ times, with the last category being $11=91-100$ times). Many of these items were previously established with regard to predictive validity and reliability (51). Data were recoded into the following categories: non-user, recreational user (one to 10 times in a year), multiple user of one substance ( $>10$ times in a year) with or without other recreational use, and multiple multidrug user (>10 times in a year).

ANS-activity was measured using the VU- Ambulatory Monitoring System (AMS; 52). Data were analyzed with VU-AMS software and data analysis support was offered by the VU-AMS department of VU University. Three electrodes were placed on the chest to measure participant's electrocardiography (ECG), and four additional electrodes were placed on chest and back for assessment of impedance cardiography (ICG). Electrode placement was done according to the VU-AMS manual (http://www.vu-ams.nl/support/instruction-manual/). Baseline functioning was measured during a 5-minute rest protocol (aquatic video, Coral Sea Dreaming, Small World Music Inc.; conform 53). Reactivity was measured during two emotional film clips ('Mohamed', 54, 'The Champ', 55), which were counterbalanced to assert that any differences could only be attributed to differences in the film clips presented. Preceding each clip, participants viewed one minute excerpts of the aquatic video while baseline functioning was measured. Values for both film clips and baselines were averaged, then change scores were 
created subtracting baseline averages from selected target episodes of the film clips. This resulted in rest as well as reactivity measures for heart rate (HR), and measures of sympathetic (pre-ejection period/PEP) and parasympathetic (respiratory sinus arrhythmia/RSA) branches of the ANS. The emotion evocation task and data preparations are described in more detail elsewhere(56)

Saliva (at least $0.1 \mathrm{ml}$ ) for cortisol and testosterone assessment was collected using a Salivette ${ }^{\circledR}$ (Sarstedt, Nümbrecht, Germany). All saliva samples were obtained on weekdays, between 12:00 and 18:00. A day before, as well as the hour before sampling, all participants were reminded of the sampling procedure. Additionally, they were reminded not to eat, drink (with the exception of water), smoke or brush their teeth during the hour before the start of the appointment. Ten minutes before sampling, participants were asked to rinse their mouth with tap water. A member of the research team gave verbal instructions before and during sampling. After saliva collection, a member of the research team wrote down subject number, date and exact sampling time. All samples were stored in the freezer the same day. Uncentrifuged samples were stored at $-20^{\circ} \mathrm{C}$ until analysis. Analyses were performed at the Endocrinology Laboratories of the University Medical Centre Utrecht.

Cortisol in saliva was measured without extraction using an in-house competitive radio-immunoassay employing a polyclonal anti-cortisol-antibody (K7348). [1,2- $\left.{ }^{3} \mathrm{H}(\mathrm{N})\right]$-Hydrocortisone (NET396250UC, PerkinElmer) was used as a tracer. The lower limit of detection was $1.0 \mathrm{nmol} / \mathrm{L}$ and inter-assay variation was $<7 \%$ at $3.3-30 \mathrm{nmol} / \mathrm{L}(\mathrm{n}$ $=80)$. Intra-assay variation was $<4 \%(n=10)$.

Testosterone in saliva was measured in duplicate using an in-house competitive radio-immunoassay employing a polyclonal anti-testosterone-antibody (Dr. Pratt AZG 3290). [1,2,6,7- $\left.{ }^{3} \mathrm{H}\right]-$ Testosterone (NET370250UC, PerkinElmer) was used as a tracer following chromatographic verification of its purity. The lower limit of detection was $10 \mathrm{pmol} / \mathrm{L}$. Inter-assay variation was $9.1,4.3$ and 5.6\% at 95,200 and $440 \mathrm{pmol} / \mathrm{L}$ respectively ( $n=12$, LKCH SL protocol 1610). Intra-assay variation was $7.2-2.5 \%$ at $38-92 \mathrm{pmol} / \mathrm{L}$ respectively $(n=10)$.

\section{Recidivism}

Recidivism data were obtained from official records in the Judicial Documentation register of the Dutch Ministry of Justice, retrieved on July $7^{\text {th }}$ 2017. New offenses were classified as general offenses, and further subdivided into violent and non-violent offenses. General recidivism was defined as any incident (including violent offenses) 
that led to an official judicial conviction, excluding technical breaches of order. Violence was defined as any (attempted) act intended to cause physical or psychological harm to others. Recidivism data were coded for the 12 months after release. The Dutch Scientific Research and Documentation center advises a data-collection timeframe of 24 months for coding official recidivism data for a 12 month follow-up time of official reconvictions, in order to ensure that criminal cases are fully closed and registered on the criminal records. When a shorter timeframe is used, it is more likely that committed offenses have not yet been registered. In the current study an 18 month timeframe was used, which may result in somewhat less certainty regarding the reconviction data collected for the 12 month follow-up time.

\section{Procedure}

Juveniles were assessed individually in a test room inside the institution. Research staff were trained with regard to electrode placement and procedures of the tasks, and remained in the room for the entire period of testing. They followed a detailed written protocol including verbal instructions. Electrodes were placed on the juveniles' chest, back, and fingers, and then connected to the VU-AMS device. Participants were instructed to sit still, and asked not to touch the electrodes. During the next ten minutes participants were asked to complete questionnaires on the computer to allow them to acclimate to the setting. During this period ANS parameters were measured as a natural baseline (acclimation period). After that, HR, HRV/RSA, and PEP were measured while juveniles completed tasks on the computer. These tasks consisted of a rest measure during a 5-minute resting protocol, a countdown task (conform 57) and the viewing of two film clips, interspersed with 1 minute baselines. In case of having problems with sitting still, a gentle reminder was provided. After completion of the ANS measurements, participants were disconnected from the VU-AMS device and asked to collect saliva in a plastic tube. Then they continued with questionnaires and tasks on the computer for the remainder of the session. The total session lasted approximately 90 minutes. The participants were compensated for their time with a €5 stipend.

\section{Statistical analyses}

Descriptive analyses were performed, and differences between the subgroups were examined using SPSS (Statistical Package for the Social Sciences, version 22). Subgroups were constructed consisting of different 
psychosocial and neurobiological measures using latent class regression analysis (Latent GOLD 4.0; 58). A latent class regression analysis (LCRA) was performed because:

1. Using LCRA we can form classes with predictive value for reoffending in one model. This differs from the classic LCA analyses, where you first form classes and then test whether they predict reoffending, with the risk that the classes are not related to reoffending, or do not differ in reoffending risk.

2. Using LCRA we can form classes based on psychosocial factors and then incorporate neurobiological predictors within those classes in the same model. This has the advantage that you can take into account that the influence of neurobiological factors may differ within the psychosocial classes.

3. Based on the LCRA model a 'tool' for calculating class membership can be created (see below), which can be used for internal and external validation, but also for calculating re-offending risk for 'new' cases.

The latent class regression model is shown in equation 1 in which $\mathrm{y}$ is the dependent variable reoffending after 12 months (no offending, non-violent offending, violent offending), $\boldsymbol{Z}^{c}$ are the psychosocial covariates (age, SES, ethnicity, psychopathic traits, problem behavior, criminal friends, substance use, and treatment motivation) and $\mathbf{z}^{p}$ the neurobiological predictors (HR reactivity, PEP reactivity, RSA reactivity, cortisol and testosterone).

$$
f\left(y \mid \mathbf{z}^{c}, \mathbf{z}^{p}\right)=\sum_{x} \pi\left(x \mid \mathbf{z}^{c}\right) f\left(y \mid x, \mathbf{z}^{p}\right)
$$

Latent class regression models were fitted for one through three classes. We evaluated the relative fit using the Bayesian Information Criterion (BIC) and the Aikake Information Criterion (AIC) (Vrieze (59).

Internal validation of the model was done by evaluating the classification fit measures. In addition, an external validation was performed to evaluate the functioning and stability of the model. This was done by predicting class membership and recidivism in 76 participants who were not included in the dataset that was used to estimate the model parameters, due to too short follow-up periods. The class membership as well as the predicted reoffending category (no, non-violent, violent) was calculated using the research prototype tool provided on the website https://architecta.shinyapps.io/PredictingYouthReoffending/. The predicted 
reoffending category was compared to the observed reoffending category. Sensitivity, specificity, positive predictive value, and negative predictive value were calculated for the reoffending categories. This should be considered as a first step in external validation, as the follow-up window is relatively short and widely varies between the 76 participants.

\section{Results}

Table 1 shows five information criteria measures which evaluate the relative fit of the model and penalize for the number of parameters ${ }^{1}$. The lower these fit measures, the better the model in terms of relative fit and parsimony. Unfortunately, although a very common result for latent class models, the different information criteria lead to a different number of classes. The BIC and CAIC would result in the 1 class model, while the AIC, AIC3 and the SABIC lead to 3 classes. The classification errors (CE) show that the percentage of classification errors is very low, $2 \%$ for the three class model, and the interpretation of this model shows that the 3 class solution could well be interpreted. The class sizes were substantial for all three classes $(.62, .21$ and .17 respectively). It was therefore decided to choose the three class model (see also Table 2).

Table 1. Information criteria of the model fit for the one, two and three class models resulting from latent class regression analysis for categories of offending within 12 months after detention

\begin{tabular}{|c|c|c|c|c|c|c|c|c|c|c|c|}
\hline & Npar & $\mathrm{L}^{2}$ & $B I C\left(L^{2}\right)$ & $\operatorname{AIC}\left(L^{2}\right)$ & $\operatorname{AIC3}\left(L^{2}\right)$ & $\operatorname{CAIC}\left(L^{2}\right)$ & SABIC $\left(L^{2}\right)$ & df & $p$ & CE & $R^{2}$ \\
\hline 1 class & 18 & 383.92 & -724.55 & -26.08 & -231.08 & -929.55 & $-7.49 \mathrm{E}+01$ & 205 & $5.30 \mathrm{E}-13$ & 0 & 0.03 \\
\hline 2 class & 54 & 268.71 & -645.10 & -69.29 & -238.29 & -814.10 & $-1.10 E+02$ & 169 & $1.60 \mathrm{E}-06$ & 0.01 & 0.38 \\
\hline 3 class & 90 & 154.03 & -565.13 & -111.97 & -244.97 & -698.13 & -143.63 & 133 & 0.1 & 0.02 & 0.73 \\
\hline
\end{tabular}

Descriptive statistics for the different classes are displayed in Table 2. An overview with descriptive statistics of neurobiological variables per recidivism type (no, non-violent, violent) is presented in Table B in the addendum. Because the latent class regression analysis is an exploratory analysis and our aim is mainly to describe relevant patterns in the data we decided to be a bit more lenient with respect to significance levels by using a type one error rate of .07 instead of the commonly used .05 . In order to interpret the classes we evaluated the effect of the covariates that predicted class membership. Figure 1 gives a graphic display of the three classes (see also

\footnotetext{
${ }^{1}$ Because of sparse frequency tables the $p$-value associated with the Chi square statistic cannot be trusted. Therefore, relative fit measures were used instead of absolute fit measures.
} 
Table $A$ in addendum for an overview of the effect of psychosocial covariates). All comparisons in the following section concern comparisons between the subgroups.

Table 2. Descriptive statistics (percentages of categorical variables, means and standard deviations of continuous variables) for the three subgroups*

\begin{tabular}{|c|c|c|c|c|c|c|}
\hline & \multicolumn{2}{|c|}{$\begin{array}{c}\text { 'Low risk - } \\
\text { psychopathic traits' } \\
(\mathrm{N}=89) \text { class } 3 \\
\%\end{array}$} & \multicolumn{2}{|c|}{$\begin{array}{l}\text { 'Medium risk - } \\
\text { adverse } \\
\text { environment' } \\
\text { (N = 23) class } 1\end{array}$} & \multicolumn{2}{|c|}{$\begin{array}{c}\text { 'High risk - } \\
\text { externalizing' } \\
\text { (N = 19) class } 2\end{array}$} \\
\hline \multicolumn{7}{|l|}{ Etnicity } \\
\hline Dutch & 28.1 & & 26.1 & & 36.8 & \\
\hline Western & 3.4 & & 0.0 & & 10.5 & \\
\hline Non-western & 68.5 & & 73.9 & & 52.6 & \\
\hline \multicolumn{7}{|l|}{ Socioeconomic status } \\
\hline Low & 36.0 & & 47.8 & & 15.8 & \\
\hline Middle & 60.7 & & 52.2 & & 63.2 & \\
\hline High & 3.4 & & 0.0 & & 21.1 & \\
\hline \multicolumn{7}{|l|}{ Criminal friends } \\
\hline Yes & 66.3 & & 95.7 & & 57.9 & \\
\hline No & 33.7 & & 4.3 & & 42.1 & \\
\hline \multicolumn{7}{|l|}{ Substance use } \\
\hline Non-user & 15.7 & & 0.0 & & 10.5 & \\
\hline Recreational user & 23.6 & & 13.0 & & 0.0 & \\
\hline Multiple user 1 substance & 24.7 & & 78.3 & & 36.8 & \\
\hline \multirow[t]{2}{*}{ Multiple user $>1$ substance } & 36.0 & & 8.7 & & 52.6 & \\
\hline & $M$ & $S D$ & $M$ & $S D$ & $M$ & $S D$ \\
\hline Age & 18.61 & 1.66 & 18.20 & 1.63 & 18.84 & 1.85 \\
\hline PT Interpersonal dimension & 10.43 & 3.71 & 9.96 & 2.92 & 12.42 & 3.50 \\
\hline PT Affective dimension & 10.53 & 3.20 & 8.39 & 2.06 & 11.68 & 3.25 \\
\hline PT Behavior dimension & 12.56 & 3.47 & 11.17 & 3.21 & 12.95 & 3.14 \\
\hline Internalizing problems & 1.28 & 1.62 & 0.78 & 1.17 & 1.42 & 2.12 \\
\hline Externalizing problems & 1.89 & 1.63 & 2.61 & 1.95 & 4.05 & 2.82 \\
\hline Attention problems & 3.48 & 2.36 & 3.39 & 2.74 & 3.11 & 1.49 \\
\hline Treatment motivation & 26.98 & 6.86 & 26.65 & 6.87 & 20.89 & 5.35 \\
\hline Trauma & 39.92 & 7.88 & 40.65 & 9.64 & 48.26 & 18.10 \\
\hline HR rest & 71.09 & 9.73 & 75.69 & 14.49 & 70.17 & 9.33 \\
\hline PEP rest & 98.26 & 19.94 & 96.92 & 21.91 & 98.06 & 20.38 \\
\hline Lg RSA rest & 1.83 & 0.23 & 1.77 & 0.25 & 1.87 & 0.19 \\
\hline HR reactivity & -3.21 & 2.47 & -4.06 & 3.31 & -3.16 & 3.00 \\
\hline PEP reactivity & 0.55 & 2.92 & -0.41 & 2.58 & -0.94 & 4.16 \\
\hline RSA reactivity & -6.88 & 19.39 & -0.83 & 16.91 & -3.10 & 15.49 \\
\hline Cortisol & 9.96 & 3.66 & 9.79 & 2.45 & 8.71 & 2.53 \\
\hline Testosterone & 284.17 & 75.63 & 264.87 & 55.11 & 292.95 & 74.13 \\
\hline
\end{tabular}

Note. $\mathrm{PT}=$ psychopathic traits; $\mathrm{HR}=$ heart rate; $\mathrm{PEP}=$ pre-ejection period; $\mathrm{Lg}=$ transformed logarithmically; RSA = respiratory sinus arrhythmia

*Please note that while in latent class analysis missing values on predictor variables are possible, for the purpose of providing descriptive statistics individuals with missing data on predictor variables cannot be 
assigned to one of the subgroups. Therefore, the $\mathrm{N}$ in Table 2 is smaller than the $\mathrm{N}$ used in analyses. The displayed numbers in the table therefore only represent an approximation.

In the 'Low risk - psychopathic traits' class, the adolescents were on average older and were not often of Dutch descent. They had higher scores on the affective and behavioral dimensions of psychopathic traits (YPIs). The juveniles scored lower on the interpersonal dimension of psychopathic traits (YPI-s), externalizing problems (BPM-Y), and trauma (CTQ-S). Few people reported multiple use of a substance. The probability of not reoffending within 12 months was .81 , and the probability of reoffending was .14 for non-violent and .05 for violent reoffending. The 'Low risk - psychopathic traits' subgroup had a class size proportion $\left(\pi_{x}\right)$ of .62. This means that $62 \%$ of the participants in the sample would be assigned to this class, or, generalized to the population, that $62 \%$ of the population would be assigned to this class.

In the 'Medium risk - adverse environment' class, the adolescents were on average younger and of non-western descent. They more often had a low or middle SES and more often reported having criminal friends. Individuals in this group scored low on the affective and behavior dimension of psychopathic traits (YPI-s) and internalizing problems (BPM-y). On average juveniles scored higher on attention problems (BPM-y) and trauma (CTQ-s). Individuals had a higher probability of being a recreational user and scored higher on treatment motivation. The probability of not reoffending was .45 , with a probability of .31 for non-violent and .24 for violent reoffending. The 'Medium risk - adverse environment' subgroup had a class size proportion of .21.

Juveniles in the 'High risk - externalizing' class on average had a high SES, were of Dutch descent and more often reported not having criminal friends. Individuals scored higher on the interpersonal dimension of psychopathic traits (YPI-s) and externalizing problems (BPM-y). They more often reported having few attention problems (BPM-y), and had a higher probability of being a non-user. Furthermore, they scored lower on treatment motivation. The probability of not reoffending was .27 , with a probability of .43 for non-violent and .30 for violent reoffending. The ‘High risk - externalizing’ subgroup had a class size proportion of .17.

Insert Figure 1 about here 
Figure 1. Subgroups of juvenile offenders (Z-scores $>1.80$ ) with different relationships to the categories of reoffending behavior (no offending, non-violent offending and violent offending) within 12 months after release. Note. PT = psychopathic traits.

\section{Neurobiological predictors}

Furthermore, we interpreted the regression weights of the neurobiological predictors within the classes. Descriptive statistics for the neurobiological parameters in the different classes are displayed in Table 2. Figure 2 gives a graphic display of the three classes (see also Table B in addendum for an overview of the effect of neurobiological predictors).

In the 'Low risk - psychopathic traits' class, there was a negative regression weight of HR reactivity (Z $=-1.86)$, and positive regression weights for cortisol $(Z=2.18)$ and PEP in rest $(Z=1.85)$, indicating that a lower HR reactivity and higher cortisol and PEP in rest were accompanied by a higher probability of not reoffending within 12 months. Lower testosterone $(Z=-2.05)$ was accompanied by a higher probability of non-violent reoffending. Lower PEP in rest $(Z=-1.82)$ and cortisol level $(Z=-1.96)$, and higher testosterone level $(Z=1.89)$, was accompanied by a higher probability of violent reoffending.

In the 'Medium risk - adverse environment' class, higher HR and RSA in rest $(Z=1.98$ and 2.22 respectively), higher HR reactivity $(Z=2.26)$, and lower cortisol level $(Z=-1.99)$ were accompanied by a higher probability of not reoffending. For non-violent reoffending, lower HR and RSA in rest $(Z=1.86$ and -1.88 respectively), and higher PEP reactivity and cortisol level $(Z=2.27$ and 2.10 respectively) were accompanied with a higher probability of non-violent reoffending. Lower PEP and RSA reactivity $(Z=-2.49$ and -2.01 respectively) and higher PEP in rest $(Z=2.23)$ were associated with a higher probability of violent reoffending.

Finally, in the 'High risk - externalizing' class, a higher PEP in rest $(Z=1.85)$ and HR reactivity $(Z=2.09)$, and a lower PEP reactivity $(Z=-1.88)$, RSA reactivity $(Z=-2.19)$, and testosterone level $(Z=-2.17)$ were accompanied by a higher probability of not reoffending. For non-violent reoffending, a higher HR in rest $(Z=$ 1.89), PEP reactivity $(Z=2.01)$, and testosterone level $(Z=2.32)$, and a lower cortisol level $(Z=-2.19)$ were accompanied by a higher probability of non-violent reoffending. Lower HR in rest $(Z=-2.25)$ and HR reactivity $(Z$ $=-2.38)$, and higher RSA reactivity $(Z=2.31)$ were accompanied by a higher probability of violent reoffending. 


\section{Insert figure 2 about here}

Figure 2. Neurobiological profiles for the three subgroups of juvenile offenders with different categories of reoffending (no offending, non-violent offending and violent offending) within 12 months after release

\section{External validation of the latent class regression model}

The data of the 76 participants with insufficient follow-up duration for the LCRA analysis was used as a reference sample to evaluate the external validity of the latent class regression model. The class membership as well as the predicted reoffending category (no, non-violent, violent) was calculated using the research tool provided on the website https://architecta.shinyapps.io/PredictingYouthReoffending/. The predicted reoffending category was compared to the observed reoffending category. The results of this analysis are presented in Table 3. For $92.1 \%$ ( $n=70$ ) of the participants, the assignment to a class and prediction of reoffending was correct. Sensitivity, specificity, positive predictive value (PPV), and negative predictive value (NPV) were calculated for the reoffending categories (see Table 4).

Table 3. Observed and predicted counts of reoffending (no, non-violent, violent) for participants with insufficient follow-up duration*

\begin{tabular}{lrrrr} 
& \multicolumn{3}{c}{ Predicted reoffending } & \\
Observed reoffending & No & Non-violent & Violent & Total \\
\hline No & 51 & 1 & 1 & 53 \\
Non-violent & 4 & 13 & & 17 \\
Violent & & & 6 & 6 \\
Total & 55 & 14 & 7 & 76 \\
\hline
\end{tabular}

* Considering the short follow-up duration, these data should be interpreted with caution

Table 4. Probabilities of correct prediction of reoffending type (sensitivity, specificity, positive predictive value (PPV) and negative predictive value (NPV))

\begin{tabular}{lrrr} 
& \multicolumn{3}{c}{ Reoffending } \\
& No & Non-violent & Violent \\
\hline Sensitivity & 0.93 & 0.93 & 0.86 \\
Specificity & 0.90 & 0.94 & 1.00 \\
PPV & 0.96 & 0.76 & 1.00 \\
NPV & 0.83 & 0.98 & 0.99 \\
\hline
\end{tabular}




\section{Discussion}

The present study aimed to use a biopsychosocial model to identify clinically relevant subgroups of juvenile offenders through latent class regression analysis. The motivation for this venture was to test whether this approach is useful to develop an integrated model of neurobiological and psychosocial risk factors to improve predictive models of reoffending, as well as to equip clinicians within juvenile justice institutions with a method to assign individuals to relevant subgroups for reoffending risk, which could subsequently inform treatment. Based on psychosocial and neurobiological characteristics of 263 juvenile offenders, three relevant subgroups in relation to reoffending behavior were identified: a 'low risk - psychopathic traits' subgroup, a 'medium risk adverse environment' subgroup, and a 'high risk - externalizing' subgroup, for which relationships between neurobiological factors and type of reoffending differed. Both psychological, social and neurobiological factors contributed to the distinction between these subgroups. The three class model could accurately predict the latent class of juveniles, both internally for the targeted population (98\%), as well as externally for new juveniles (92\%). The model showed good accuracy in terms of sensitivity, specificity, and positive and negative predictive value.

Psychosocial offender characteristics that proved to be important in the current study, also proved significant for allocation to subgroups in earlier research, such as previous offending behavior (12), problem behavior, psychopathic traits, and substance use $(2,6,9-11,15)$. Individuals in the 'Low risk - psychopathic traits' subgroup were characterized by the least risk factors. They were somewhat older, showed higher affective and behavioral psychopathic traits, but less externalizing behavior. The finding of psychopathic traits in the low risk group is in contrast with most studies showing high risks of recidivism in adolescents with psychopathic traits (for a metaanalysis: (60). However, the YPI has previously shown to be less predictive (61) and predictive value is also lower for older subjects and for formal recidivism, maybe reflecting an underestimation in recidivism for this group (60). Individuals in the 'Medium risk - adverse environment' subgroup more often presented with trauma and attentional problems. This subgroup appears to be characterized by poorer environmental conditions: being more likely to come from a disadvantageous neighbourhood, having criminal friends, and using substances recreationally. Individuals in the 'High risk - externalizing' subgroup were characterized by relatively positive 
environmental circumstances, but high externalizing problems and interpersonal psychopathic traits. This is in concordance with previous studies where individual factors are more strongly related to recidivism as opposed to only environmental mental health factors. Such findings are explained by the "social push" hypothesis (62) which argues that where an antisocial child lacks social factors that push or predispose them to antisocial behaviour, then neurobiological risk factors more likely explain antisocial behaviour. However, it contradicts studies where the combination of environmental and individual mental health factors usually leads to highest risk of reoffending (9) (63)

By using the LCRA approach the neurobiological parameters initially contributed to the formation of the three classes. Although the neurobiological differences between the subgroups may not seem to have any clinical relevance in isolation, they do have a distinctive value within the prediction model. Moreover, by incorporating the neurobiological predictors within those classes, it was shown that they further differentiated within the classes which individual is at risk of no, non-violent or violent recidivism based on specific neurobiological profiles. It is important to note that with this approach, the model assesses the differential effects of neurobiological factors within each subgroup, which means that differences in neurobiological measures do not directly mean that the values reflect a high or low value of the predictor under consideration, but only refer to differences in neurobiological profiles between subgroups. This is fundamentally different from assessing neurobiological differences between delinquent juveniles and controls as was done in most previous studies, and therefore also shows a more complex picture of relationships between neurobiological measures and reoffending as compared to earlier research. This may also explain why in previous studies where neurobiological factors were related to reoffending in the total group of juvenile offenders show small and inconsistent results (De Vries-Bouw et al. 2011). Moreover, it also shows why there is currently no consensus on when biomarker levels are clinically significantly aberrant and development of cut-off values for neurobiological factors has so far been unsuccessful. This is where a research tool as discussed within the current study may be important, as it is better equipped than e.g. the clinical view at differentiating small differences within heterogeneous groups of already delinquent juveniles. This study underlines that the importance lies not in establishing cut-offs, but in determining which variables provide the most information, especially when trying to establish reoffending risk in groups of severely delinquent juveniles. The main advantage of the current approach is that, even though the model is quite complex, it can still be valuable for practical usage, because the tool can handle continuous values 
of neurobiological measures, which means there is no need for establishing strict cut-off values or clinical interpretation of neurobiological profiles.

However, some neurobiological factors emerge more consistently than others. Several of the relationships found in the current study correspond to relationships between neurobiological measures and reoffending from earlier research. For both groups with higher (violent) reoffending risk for example, parasympathetic nervous system (PNS; or RSA) reactivity comes forward as an important factor for violent reoffending. Within the "High risk externalizing" subgroup it was a somewhat higher, and within the "Medium risk - adverse environment" subgroup lower PNS reactivity that was associated with violent reoffending. Aberrant PNS reactivity was previously found to be predictive of reoffending $(27,29)$. Furthermore, HR weighed in the allocation to risk subgroups. The finding that a lower HR, both at rest and reactivity, is associated with (violent) reoffending (in the “High risk - externalizing" subgroup) is also conform previous findings (25-28). In more serious risk groups, these two biological markers, HR and PNS (re)activity, therefore seem relevant. They appear to be in the forefront among juveniles who commit (serious) recidivism.

\section{Study limitations}

It is important to note that the described model should be considered as a preliminary model in a specific subgroup of delinquent juveniles. First, the current model only applies to the group of delinquent juveniles from juvenile justice institutions that are already severely delinquent and at high risk of reoffending. It cannot as a matter of course be argued that the links between the factors determining the subgroups and reoffending can be generalized to risk factors for delinquent behavior in the general population. However, the results can support existing ideas and theories on risk taxation for (violent) reoffending. The current study using LCRA analyses resulted in finding informative subgroups with different risks of reoffending. Within juvenile justice institutions, assessment of the risk for reoffending is an important issue, and this model, although it should be replicated in a separate sample, provides a first step to improve current risk assessment by including neurobiological factors as well. Second, it was not possible to enter all characteristics that were collected under the current project in the analyses in view of statistical power. Moreover, it is likely that other potentially important information that we are not yet aware of was not collected in the current study. Furthermore, the ratio of sample size to number of parameters did not allow to examine the fit of models with more than three classes. Adding other information 
could possibly change the composition of the subgroups. Third, it is important to underline that questionnaires in this study were self-report measures. For future studies, a combination of information from different sources may be desirable (e.g. a combination of self-report and other report). Should juveniles in clinical practice prove to be reluctant to provide information, information from other sources may offer a helpful solution. Finally, a follow-up period of 12 months for recidivism data is relatively short to allow for firm statements. Therefore, follow-up studies with a longer follow-up duration are necessary. If the follow-up duration is relatively short, there is a possibility that individuals will be acquitted (on appeal), or that offences that were committed were in fact not yet processed at the time of the recidivism request.

\section{Implications for clinical practice}

The current study offers a first attempt to provide a new approach to increase the insight into biopsychosocial profiles of juvenile offenders and reoffending. The approach appears useful to develop an integrated model of neurobiological and psychosocial risk factors to improve predictive models of reoffending, as well as to equip clinical practice with a method to assign individuals to relevant subgroups for reoffending risk, which could subsequently inform treatment. This seems a promising step for integrating neurobiological risk factors in clinical practice. To facilitate practical use, a research tool prototype was constructed for the current study that could be developed into a clinical tool for professionals in the future. The research tool prototype correctly classified a large proportion of juveniles to one of the distinguished subgroups, showing both good internal and external validity of the three subgroups. At the moment, however, the research tool is still a preliminary model in a relatively small sample of juvenile delinquents and should be further developed and validated. Further research and replication of the findings are necessary, at the least with a larger sample, before the tool can be used to inform treatment of young offenders. Furthermore, at present the current prototype is not capable of dealing with missing information; a tool that is capable of this is under development. Future studies will have to reveal whether such a tool can be helpful in clinical practice. 


\section{Ethics approval and consent to participate}

All participants, and when under the age of 18 also parents/caregivers, signed an informed consent document before participation. This study was approved by the Ethics Committee at the University of Amsterdam, and performed in accordance with the ethical standards described in the 1964 Declaration of Helsinki.

\section{Availability of data and materials}

The datasets used and/or analysed during the current study are available from the corresponding author on reasonable request.

\section{Competing interests}

The authors state that they have no competing interests

\section{Funding}

This study was funded by the Ministry of Justice and Security of the Netherlands.

\section{Author's contributions}

ELdR collected the data, contributed to analyses and interpretation of the data, and was a major contributor in writing the manuscript

SB performed the LCRA analyses and was a major contributor in writing the manuscript, specifically regarding analyses and interpretation of the results

AP designed the initial project, supervised the project and contributed to writing the manuscript $\mathrm{RV}$ supervised the project and contributed to interpretation of the data and writing the manuscript LvD supervised the project and contributed to interpretation of the data and writing the manuscript LMCJ contributed to the design of the study, supervised the project, contributed to the analyses and interpretation of data and was a major contributor in writing the manuscript.

\section{Acknowledgements}

This work was supported by the Academic Workplace Risk Youth (AWRJ). We thank the juveniles and staff of the participating institutions; H.S. van der Baan, R. W. Wiers, A. Collot D'Escury, B. Verschuere, and M.D. de Boer, department of Developmental Psychology, University of Amsterdam, for their cooperation in data collection; 
Laboratory of Clinical Chemistry \& Haematology (LKCH), University Medical Centre Utrecht, for performing hormone analyses. The authors report no potential conflicts of interest.

\section{References}

1. Colins O, Vermeiren R, Schuyten G, Broekaert E. Psychiatric Disorders in Property, Violent, and Versatile Offending Detained Male Adolescents. American Journal of Orthopsychiatry. 2009;79(1):31-8.

2. Decuyper M, Colins OF, De Clercq B, Vermeiren R, Broekaert E, Bijttebier P, et al. Latent personality profiles and the relations with psychopathology and psychopathic traits in detained adolescents. Child Psychiatry Hum Dev. 2013;44(2):217-32.

3. Wartna BSJ, Tollenaar N, Verweij S, Alberda DL, Essers AAM. Recidivebericht 2015; Landelijke cijfers over de terugval van justitiabelen bestraft in de periode 2002-2012, Factsheet 2016-1. . The Hague, The Netherlands: WODC. ; 2016.

4. Chng GS, Chu CM, Zeng G, Li D, Ting MH. A Latent Class Analysis of Family Characteristics Linked to Youth Offending Outcomes. Journal of Research in Crime and Delinquency. 2016;53(6):765-87.

5. Dembo R, Briones-Robinson R, Ungaro R, Karas L, Gulledge L, Greenbaum PE, et al. Problem Profiles of at-Risk Youth in Two Service Programs: A Multi-Group, Exploratory Latent Class Analysis. Criminal Justice and Behavior. 2011;38(10):988-1008.

6. Schwalbe CS, Macy RJ, Day SH, Fraser MW. Classifying Offenders An Application of Latent Class Analysis to Needs Assessment in Juvenile Justice. Youth Violence and Juvenile Justice. 2008;6(3):279-94.

7. Stefurak T, Calhoun GB. Subtypes of female juvenile offenders: a cluster analysis of the Millon Adolescent Clinical Inventory. Int J Law Psychiatry. 2007;30(2):95-111.

8. Stefurak T, Calhoun GB, Glaser BA. Personality typologies of male juvenile offenders using a cluster analysis of the Millon Adolescent Clinical Inventory introduction. Int J Offender Ther Comp Criminol. 2004;48(1):96-110.

9. Geluk CAM, van Domburgh L, Doreleijers TAH, Jansen LMC, Bouwmeester S, Garre FG, et al. Identifying children at risk of problematic development: Latent clusters among childhood arrestees. J Abnorm Child Psychol. 2014;42(4):669-80.

10. Wareham J, Dembo R, Poythress NG, Childs K, Schmeidler J. A latent class factor approach to identifying subtypes of juvenile diversion youths based on psychopathic features. Behav Sci Law. 2009;27(1):71-95.

11. Dembo R, Wareham J, Poythress NG, Meyers K, Schmeidler J. Psychosocial Functioning Problems Over Time Among High-Risk Youths A Latent Class Transition Analysis. Crime \& Delinquency. 2008;54(4):644-70.

12. Mulder E, Vermunt J, Brand E, Bullens R, van Marle H. Recidivism in subgroups of serious juvenile offenders: different profiles, different risks? Criminal Behavior and Mental Health. 2012;22(2):122-35.

13. Vincent GM, Vitacco MJ, Grisso T, Corrado RR. Subtypes of adolescent offenders: affective traits and antisocial behavior patterns. Behav Sci Law. 2003;21(6):695-712.

14. Baglivio MT, Jackowski K, Greenwald MA, Howell JC. Serious, violent, and chronic juvenile offenders: A statewide analysis of prevalence and prediction of subsequent recidivism using risk and protective factors.

Criminology \& Public Policy. 2014;13(1):83-116.

15. Hillege SL, Brand EF, Mulder EA, Vermeiren RRJM, van Domburgh L. Serious juvenile offenders: classification into subgroups based on static and dynamic charateristics. Child Adolesc Psychiatry Ment Health. 2017;11(1):67.

16. Popma A, Raine A. Will future forensic assessment be neurobiologic? Child Adolesc Psychiatric Clin N Am. 2006;15(2): 429-44.

17. Beauchaine TP, Neuhaus E, Brenner SL, Gatzke-Kopp L. Ten good reasons to consider biological processes in prevention and intervention research. Development and psychopathology. 2008;20(3):745-74.

18. Glenn AL. Using biological factors to individualize interventions for youth with conduct problems: current state and ethical issues. International journal of law and psychiatry. 2018.

19. Hinnant JB, Erath SA, El-Sheikh M. Harsh parenting, parasympathetic activity, and development of delinquency and substance use. Journal of abnormal psychology. 2015;124(1):137. 
20. Farrington DP. The relationship between low resting heart rate and violence. In A. Raine, P. A. Brennan, D. P. Farrington \& S. A. Mednick (Eds.), Biosocial bases of violence (pp. 89-106). . New York: Plenum Press. 1997.

21. Rocque $M$, Welsh BC, Raine A. Biosocial criminology and modern crime prevention. Journal of Criminal Justice. 2012;40(4):306-12.

22. Ortiz J, Raine A. Heart rate level and antisocial behavior in children and adolescents: A meta-analysis. Journal of the American Academy of Child \& Adolescent Psychiatry. 2004;43(2):154-62.

23. Portnoy J, Farrington DP. Resting heart rate and antisocial behavior: An updated systematic review and meta-analysis. Aggression and violent behavior. 2015;22:33-45.

24. Beauchaine TP. Respiratory sinus arrhythmia: A transdiagnostic biomarker of emotion dysregulation and psychopathology. Current opinion in psychology. 2015;3:43-7.

25. Raine A, Venables PH, Williams M. Relationships between central and autonomic measures of arousal at age 15 years and criminality at age 24 years. Archives of General Psychiatry. 1990;47(11):1003-7.

26. Raine A, Venables PH, Williams M. High autonomic arousal and electrodermal orienting at age 15 years as protective factors against criminal behavior at age 29 years. The American journal of psychiatry. 1995;152(11):1595-600.

27. De Vries-Bouw M, Popma A, Vermeiren R, Doreleijers TA, Van De Ven PM, Jansen LM. The predictive value of low heart rate and heart rate variability during stress for reoffending in delinquent male adolescents. Psychophysiology. 2011;48(11):1597-603.

28. Jennings WG, Piquero AR, Farrington DP. Does resting heart rate at age 18 distinguish general and violent offending up to age 50? Findings from the Cambridge Study in Delinquent Development. Journal of Criminal Justice. 2013;41(4):213-9.

29. De Ruigh EL, Kleeven ATH, Jansen LMC, De Vries Robbé M, Vermeiren RRJM, Mulder EA, et al. Predicting youth reoffending after incarceration: added value of protective factors and RSA reactivity on risk assessment.

30. El-Sheikh M, Hinnant JB. Marital conflict, respiratory sinus arrhythmia, and allostatic load: Interrelations and associations with the development of children's externalizing behavior. Development and psychopathology. 2011;23(3):815-29.

31. Gao Y, Raine A, Venables PH, Dawson ME, Mednick SA. Association of poor childhood fear conditioning and adult crime. American Journal of Psychiatry. 2009;167(1):56-60.

32. Hinnant JB, Erath SA, Tu KM, El-Sheikh M. Permissive parenting, deviant peer affiliations, and delinquent behavior in adolescence: The moderating role of sympathetic nervous system reactivity. Journal of abnormal child psychology. 2016;44(6):1071-81.

33. Rosell DR, Siever LJ. The neurobiology of aggression and violence. CNS spectrums. 2015;20(3):254-79.

34. Platje E, Jansen LM, Raine A, Branje SJ, Doreleijers TA, de Vries-Bouw M, et al. Longitudinal associations in adolescence between cortisol and persistent aggressive or rule-breaking behavior. Biological psychology. 2013;93(1):132-7.

35. Book AS, Starzyk KB, Quinsey VL. The relationship between testosterone and aggression: a metaanalysis. Aggression and Violent Behavior. 2001;6(6):579-99.

36. Popma A, Vermeiren R, Geluk CA, Rinne T, van den Brink W, Knol DL, et al. Cortisol moderates the relationship between testosterone and aggression in delinquent male adolescents. Biological psychiatry. 2007;61(3):405-11.

37. Moffitt TE, order ia, Arseneault L, Jaffee SR, Kim-Cohen J, Koenen KC, et al. Research review: DSM-V conduct disorder: Research needs for an evidence base. Journal of Child Psychology and Psychiatry. 2008;49(1):3-33.

38. Alink LRA, van ljzendoorn MH, Bakermans-Kranenburg MJ, Mesman J, Juffer F, Koot HM. Cortisol and externalizing behavior in children and adolescents: Mixed meta-analytic evidence for the inverse relation of basal cortisol and cortisol reactivity with externalizing behavior. Dev Psychobiol. 2008;50(5):427-50.

39. De Ruigh EL, Kleeven ATH, Jansen LMC, De Vries Robbé M, Vermeiren RRJM, Mulder EA, et al. Predicting youth reoffending after incarceration: added value of protective factors and RSA reactivity Journal of Forensic Psychiatry and Psychology 2020.

40. Bouwmeester S, Sijtsma K, Vermunt JK. Latent class regression analysis for describing cognitive developmental phenomena: An application to transitive reasoning. European Journal of Developmental Psychology. 2004;1(1):67-86. 
41. van Baardewijk Y, Andershed H, Stegge H, Nilsson KW, Scholte E, Vermeiren R. Development and tests of short versions of the youth psychopathic traits inventory and the youth psychopathic traits inventory-child version. European Journal of Psychological Assessment. 2010;26(2):122-8.

42. Colins OF, Noom M, Vanderplasschen W. Youth Psychopathic Traits Inventory-Short Version: A further test of the internal consistency and criterion validity. Journal of Psychopathology and Behavioral Assessment. 2012;34(4):476-86.

43. Vahl P, Colins OF, Lodewijks HP, Markus MT, Doreleijers TA, Vermeiren RR. Psychopathic-like traits in detained adolescents: Clinical usefulness of self-report. European child \& adolescent psychiatry. 2014;23(8):691-9.

44. Achenbach T, McConaughy S, Ivanova M, Rescorla L. Manual for the ASEBA brief problem monitor (BPM). Burlington, VT: ASEBA. 2011:1-33.

45. Achenbach T, Rescorla L. Manual for the ASEBA School-Age Forms \& Profiles. . Burlington, VT: University of Vermont, Research Center for Children, Youth, and Families.2001.

46. van Binsbergen M. Motivatie voor behandeling: Ontwikkeling van behandelmotivatie in een justitiële instelling: Garant; 2003.

47. Van der Helm G, Wissink I, De Jongh T, Stams G. Measuring treatment motivation in secure juvenile facilities. International journal of offender therapy and comparative criminology. 2013;57(8):996-1008.

48. Bernstein DP, Stein JA, Newcomb MD, Walker E, Pogge D, Ahluvalia T, et al. Development and validation of a brief screening version of the Childhood Trauma Questionnaire. Child abuse \& neglect. 2003;27(2):169-90.

49. Bernstein DP, Ahluvalia T, Pogge D, Handelsman L. Validity of the Childhood Trauma Questionnaire in an adolescent psychiatric population. Journal of the American Academy of Child \& Adolescent Psychiatry. 1997;36(3):340-8.

50. Forde DR, Baron SW, Scher CD, Stein MB. Factor structure and reliability of the childhood trauma questionnaire and prevalence estimates of trauma for male and female street youth. Journal of interpersonal violence. 2012;27(2):364-79.

51. Graham JW, Flay BR, Johnson CA, Hansen WB, Grossman L, Sobel JL. Reliability of self-report measures of drug use in prevention research: Evaluation of the Project SMART questionnaire via the test-retest reliability matrix. Journal of drug education. 1984;14(2):175-93.

52. Klaver C, De Geus E, De Vries J. Ambulatory monitoring system. In F. J. Maarse (Ed.),. Computers in psychology 5, Applications, methods, and instrumentation. 1994:254-68. Lisse, the Netherlands: Swets \& Zeitlinger.

53. Scarpa A, Haden SC, Tanaka A. Being hot-tempered: Autonomic, emotional, and behavioral distinctions between childhood reactive and proactive aggression. Biological Psychology. 2010;84(3):488-96.

54. De Wied M, van Boxtel A, Matthys W, Meeus W. Verbal, facial and autonomic responses to empathyeliciting film clips by disruptive male adolescents with high versus low callous-unemotional traits. Journal of abnormal child psychology. 2012;40(2):211-23.

55. Lovell DP, Zeffirelli FD. The champ [Motion picture]. United States: MGM/Pathe Home Video. 1979.

56. de Ruigh EL, Jansen LMC, Vermeiren R, Popma A. Psychopathic traits and empathic functioning in detained juveniles: Withdrawal response to empathic sadness. International Journal of Forensic Mental Health. 2019;18(4):336-49.

57. Wang P, Baker LA, Gao Y, Raine A, Lozano DI. Psychopathic traits and physiological responses to aversive stimuli in children aged 9-11 years. Journal of abnormal child psychology. 2012;40(5):759-69.

58. Vermunt JK, Magidson J. Latent Class Cluster Analysis. In: Hagenaars, J.A. \& Vermunt, J.K. \& Magidson, J. (2005). Latent GOLD 40 user's guide 2002:Belmont, MA: Statistical Innovations Inc.

59. Vrieze SI. Model selection and psychological theory: a discussion of the differences between the Akaike information criterion (AIC) and the Bayesian information criterion (BIC). Psychological methods. 2012;17(2):228-43.

60. Asscher JJ, van Vugt ES, Stams GJJM, Dekovic M, Eichelsheim VI, Yousfi S. The relationship between juvenile psychopathic traits, delinquency and (violent) recidivism: A meta-analysis. Journal of Child Psychology and Psychiatry. 2011;52(11):1134-43.

61. Colins OF, Fanti KA, Andershed H, Mulder E, Salekin RT, Blokland A, et al. Psychometric properties and prognostic usefulness of the Youth Psychopathic Traits Inventory (YPI) as a component of a clinical protocol for detained youth: A multiethnic examination. Psychological Assessment. 2017;29(6):740-53.

62. Raine A. Biosocial studies of antisocial and violent behavior in children and adults: A review. Journal of Abnormal Child Psychology. 2002;30(4):311-26. 
Using the biopsychosocial model for identifying subgroups of detained juveniles at different risk of re-offending in practice: a latent class regression analysis approach

63. Mulder E, Brand E, Bullens R, van Marle H. Risk Factors for Overall Recidivism and Severity of Recidivism in Serious Juvenile Offenders. International Journal of Offender Therapy and Comparative Criminology. 2010;55(1):118-35. 
Figures

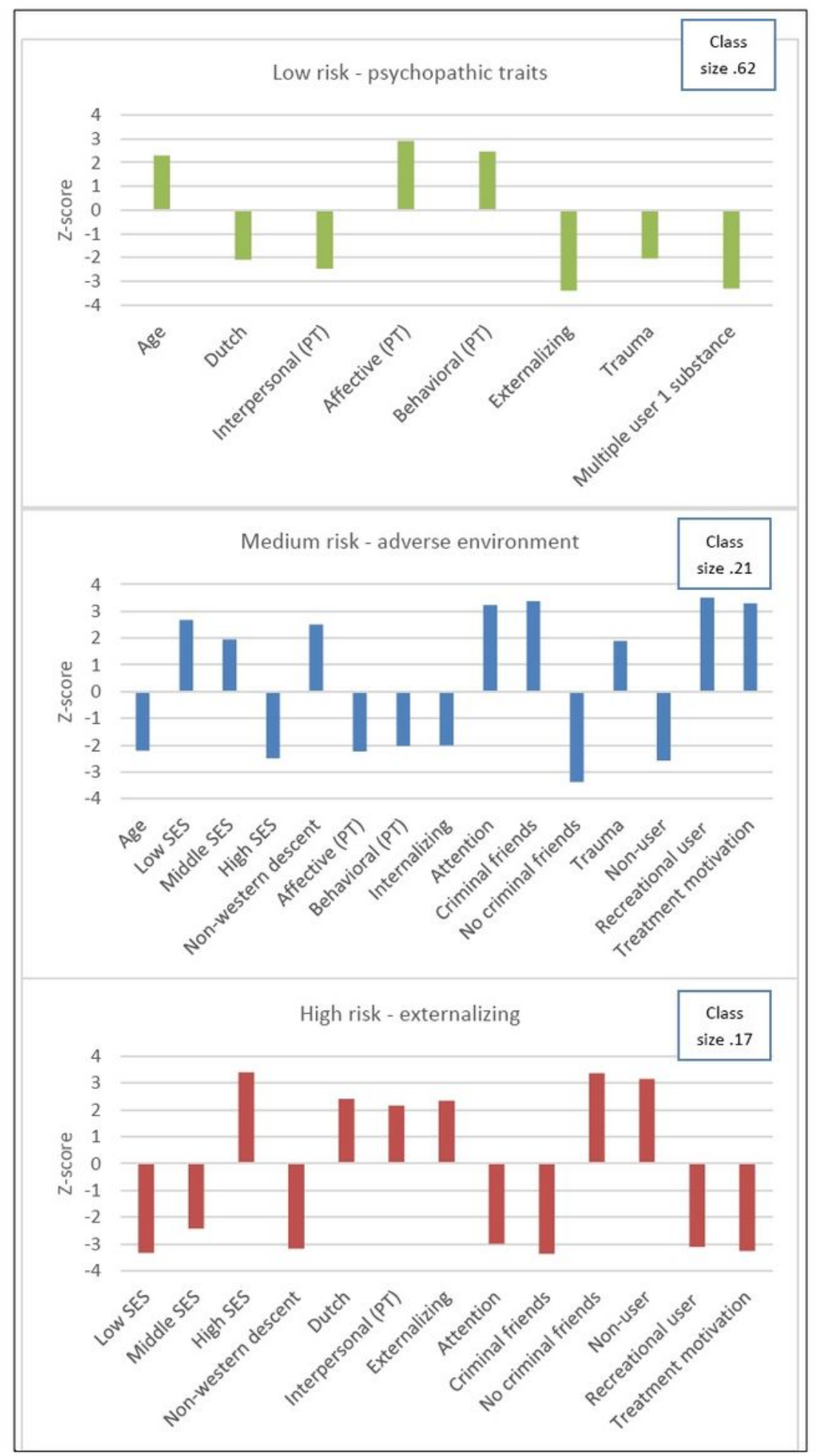

Figure 1

Subgroups of juvenile offenders (Z-scores > 1.80) with different relationships to the categories of reoffending behavior (no offending, non-violent offending and violent offending) within 12 months after release. Note. $\mathrm{PT}$ = psychopathic traits. 


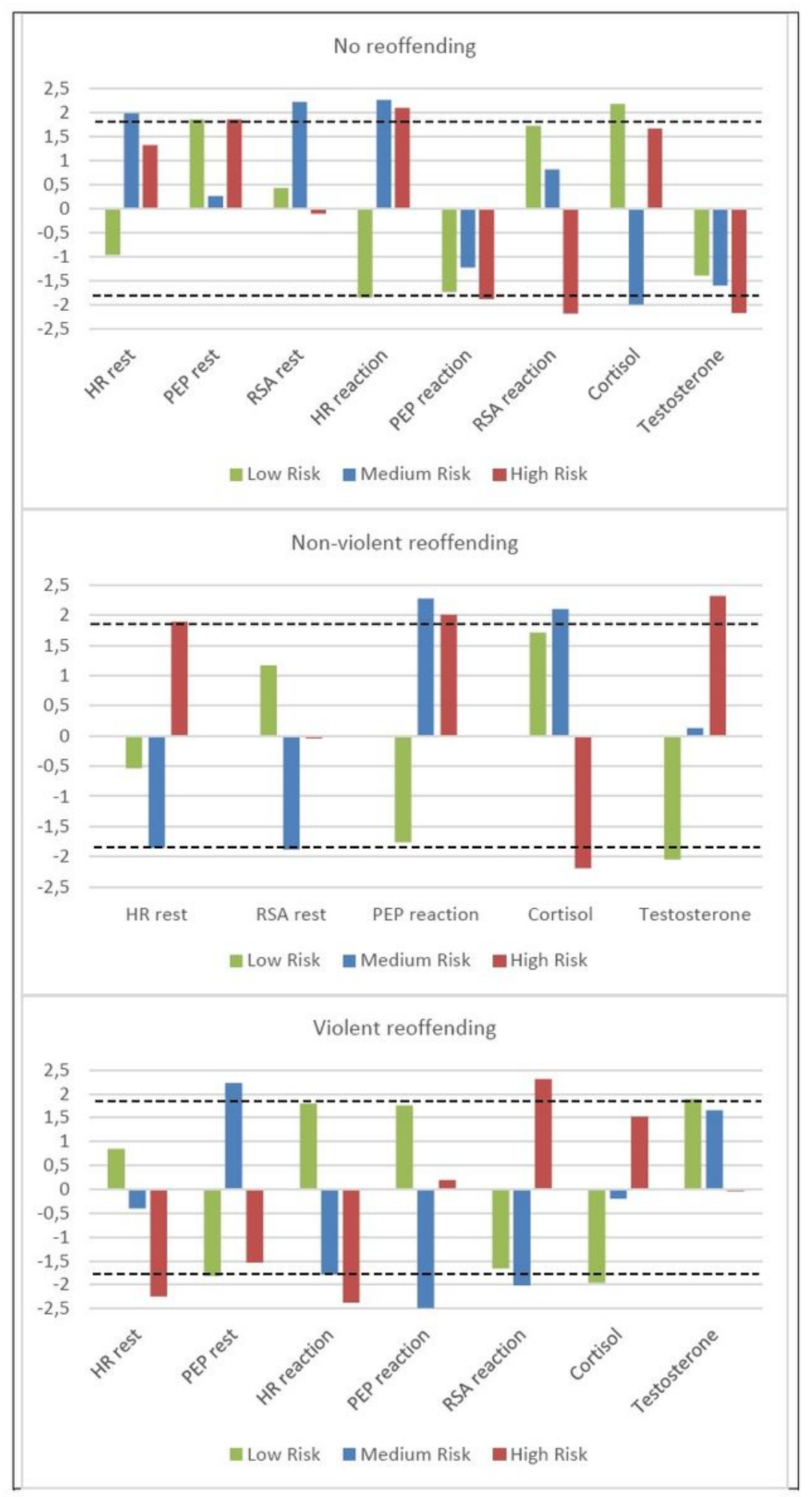

\section{Figure 2}

Neurobiological profiles for the three subgroups of juvenile offenders with different categories of reoffending (no offending, non-violent offending and violent offending) within 12 months after release

\section{Supplementary Files}


This is a list of supplementary files associated with this preprint. Click to download.

- Additionalfile1.docx 